Pacific Journal of Mathematics

SPACES OF CONSTANT PARA-HOLOMORPHIC SECTIONAL 


\section{SPACES OF CONSTANT PARA-HOLOMORPHIC SECTIONAL CURVATURE}

\section{P. M. Gadea and A. Montesinos Amilibia}

We consider the sectional curvatures for metric $\left(J^{4}=1\right)$-manifolds, and study particularly the general expression of the metric and almostproduct structure in normal coordinates for para-Kaehlerian manifolds of constant para-holomorphic sectional curvature. We also introduce models of such spaces.

1. Introduction. A metric $\left(J^{4}=1\right)$-manifold (cfr. [3], [11]) is a pseudo-Riemannian manifold $\left(M^{n}, g\right)$ together with a $(1,1)$ tensor field $J$ such that $J^{4}=1$ and whose characteristic polynomial is $(x-1)^{r_{1}}(x+1)^{r_{2}}\left(x^{2}+1\right)^{s}$ with $r_{1}+r_{2}+2 s=n$; also, the tensor fields $g$ and $J$ are related by one of the following relations:

(i) $g(J X, Y)+g(X, J Y)=0$ (then $g$ is necessarily pseudo-Riemannian and $\left.r_{1}=r_{2}\right)$;

(ii) $g$ is Riemannian and $g(J X, J Y)=g(X, Y)$.

In the first case it is said that $g$ is an aem (adapted in the electromagnetic sense metric), because this situation generalizes in a sense that of Mishra [8] and Hlavatý [4]; in the second one, $g$ is called arm (adapted Riemannian metric).

In this note we consider, $g$ being an aem, the $J$-Kaehler manifolds, that is $\left(J^{4}=1\right)$-manifolds such that $\nabla J=0$, where $\nabla$ is the LeviCivita connection of $g$, and study the $J$-sectional curvature which generalizes the usual holomorphic-type sectional curvatures. We define the spaces of constant $J$-sectional curvature, and prove a lemma of Schur type. Also, we obtain explicitly the models corresponding to the situation of an aem $g$ and $J^{2}=1$.

2. Terminology. We shall use the following terminology:

$\left(J^{4}=1\right)$-manifold: the pair $\left(M^{n}, J\right)$, where $J$ is a $(1,1)$ tensor field such that $J^{4}=1$ and whose characteristic polynomial is $(x-1)^{r_{1}}(x+1)^{r_{2}}\left(x^{2}+1\right)^{s}$ with $r_{1}+r_{2}+2 s=n$.

e-metric $\left(J^{4}=1\right)$-manifold: a $\left(J^{4}=1\right)$-manifold $\left(M^{n}, J\right)$ together with an aem, that is a pseudo-Riemannian metric $g$ such that $g(J X, Y)$ $+g(X, J Y)=0$. 
Riemannian $\left(J^{4}=1\right)$-manifold: a $\left(J^{4}=1\right)$-manifold $\left(M^{n}, J\right)$ with an arm, i.e., a Riemannian metric $g$ such that $g(J X, J Y)=g(X, Y)$.

The remaining cases have already their own names:

almost para-Hermitian manifold (see Libermann ([7]): it is an $e$ metric $\left(J^{4}=1\right)$-manifold such that $J^{2}=1$, or in other terms, $s=0$ (see also Legrand [6]).

Riemannian almost-product manifold: a Riemannian $\left(J^{4}=1\right)$-manifold with $J^{2}=1$, or equivalently $s=0$.

almost-Hermitian manifold: it is the case of $J^{2}=-1$ or equivalently $r_{1}=r_{2}=0$. In this case there is no distinction between aem and arm.

3. $J$-sectional curvature. We consider first that $(M, J, g)$ is an $e$ metric $\left(J^{4}=1\right)$-manifold. We have $g(J X, Y)+g(X, J Y)=0$. Then necessarily $r_{1}=r_{2}=r$ (see [3]). Let $\nabla$ be the Levi-Civita connection of $g$. The curvature operator $R(X, Y): \Gamma(\otimes T M) \rightarrow \Gamma(\otimes T M)$ is defined by

$$
R(X, Y)=\nabla_{[X, Y]}-\left[\nabla_{X}, \nabla_{Y}\right]
$$

and we use the following convention for the Riemann-Christoffel tensor field

$$
R(X, Y, Z, W)=g(R(X, Y) Z, W) .
$$

We shall denote also by $R$ the value of $R$ at a generic point $x \in M$. Then, if $X, Y \in T_{X} M$, we put

$$
\bar{K}(X, Y)=R(X, Y, X, Y) .
$$

A subspace $E$ of $T_{x} M$ is said to be non-degenerate if $g \mid E$ is nondegenerate. If $\{X, Y\}$ is a basis of a plane $E$ of $T_{x} M$, then $E$ is non-degenerate if and only if

$$
g(X, X) g(Y, Y)-g(X, Y)^{2} \neq 0 .
$$

For any non-degenerate plane $E$ of $T_{x} M$ we define the sectional curvature as

$$
K(X, Y)=\frac{\bar{K}(X, Y)}{g(X, X) g(Y, Y)-g(X, Y)^{2}},
$$

where $\{X, Y\}$ is any basis of $E ; K(X, Y)$ only depends on $E$.

Since $g(J X, Y)+g(X, J Y)=0$, then $g(X, J X)=0$. If $X, J X \in T_{X} M$ are linearly independent, they determine a plane of $T_{X} M$ that we call the $J$-section defined by $X$. The sectional curvature of $\{X, J X\}$ is only defined if $g(l X, l X)^{2} \neq g\left(l_{3} X, l_{3} X\right)^{2}$, where

$$
l=\frac{1}{2}\left(1+J^{2}\right), \quad l_{3}=\frac{1}{2}\left(1-J^{2}\right),
$$


are, respectively, the projectors upon the almost-product and the almost-complex subbundles of $T M$ defined by $J$. In that case we put

$$
\bar{H}(X)=\bar{K}(X, J X), \quad H(X)=K(X, J X),
$$

and say that $H(X)$ is the $J$-sectional curvature determined by $X$.

If $\nabla J=0$ we say that $(M, g, J)$ is an $e-\left(J^{4}=1\right)$-Kaehler manifold. The characterization of these manifolds is given through the following results, where we put

$$
F(X, Y)=g(X, J Y)=-F(Y, X) .
$$

3.1. Lemma. Let $(M, g, J)$ be an e-metric $\left(J^{4}=1\right)$-manifold. Then:

$$
\begin{aligned}
4 g\left(\left(\nabla_{X} J\right) Y, Z\right)= & -2 d F(X, Y, Z)+2 d F\left(X, J^{2} Y, J^{2} Z\right) \\
& +2 d F\left(J X, J Y, J^{2} Z\right)+2 d F\left(J X, J^{2} Y, J Z\right) \\
& -g\left(N(Y, Z), J^{3} X\right)+g(N(J Y, J Z), J X) \\
& +g\left(N(X, J Y), J^{2} Z\right)+g\left(N(J Z, X), J^{2} Y\right),
\end{aligned}
$$

where $N(X, Y)=2\left\{[J X, J Y]+J^{2}[X, Y]-J[J X, Y]-J[X, J Y]\right\}$ defines the Nijenhuis tensor of $J$.

Proof. We have

$$
4 g\left(\left(\nabla_{X} J\right) Y, Z\right)=4 g\left(\nabla_{X}(J Y), Z\right)+4 g\left(\nabla_{X} Y, J Z\right) ;
$$

$$
\begin{aligned}
2 g\left(\nabla_{X} Y, Z\right)= & X(g(Y, Z))+Y(g(Z, X))-Z(g(X, Y))+g([X, Y] Z) \\
& +g([Z, X], Y)+g([Z, Y], X) \\
d F(X, Y, Z)= & X(g(Y, J Z))-Y(g(X, J Z))+Z(g(X, J Y)) \\
& -g([X, Y], J Z)+g([X, Z], J Y)-g([Y, Z], J X),
\end{aligned}
$$

and our claim is obtained directly by application of these formulae.

3.2. Corollary. In an e-metric $\left(J^{4}=1\right)$-manifold $(M, J, g)$, the condition $\nabla J=0$ is equivalent to the simultaneous verification of the following conditions:

(a) $N=0$

(b) $d F=0$.

Proof. If $N=0$ and $d F=0$, it is obvious by 3.1 that $\nabla J=0$. If $\nabla J=0$, then $d F=0$, because $\nabla g=0$; also, $N=0$ as it is easily checked from the expression of $N$, having in mind that $\nabla$ is torsionless. 
If $(M, g, J)$ is an almost para-Hermitian manifold and $\nabla J=0$, we then have a hyperbolic Kaehler manifold (Raševski [10]), also called para-Kaehler manifold (Libermann [7]). See also Prvanović [9] and references therein. We adopt Libermann's terminology. The preceding result implies that an $e-\left(J^{4}=1\right)$-Kaehler manifold is locally the product of a para-Kaehler manifold and a Kaehler manifold.

3.3. Proposition. On an e-( $\left.J^{4}=1\right)$-Kaehler manifold we have

$$
R(X, Y, Z, J W)+R(X, Y, J Z, W)=0 .
$$

Proof. By applying the operator $R(X, Y)$, we have

$$
\begin{aligned}
R(X, Y)(g(Z, J W)) & =0=g(R(X, Y) Z, J W)+g(Z, R(X, Y) J W) \\
& =R(X, Y, Z, J W)-g(J Z, R(X, Y) W) \\
& =R(X, Y, Z, J W)+R(X, Y, J Z, W) .
\end{aligned}
$$

3.4. Proposition. Let $(M, J, g)$ be an e-( $\left.J^{4}=1\right)$-Kaehler manifold. Then, if $\bar{H}(X)=0$ for all $X \in T M$, we have $R=0$.

Proof. We consider the following $(0,4)$ tensor field $Q$ which generalizes that of the Kaehler case (see [5]):

$$
Q(X, Y, Z, W)=R(X, J Y, Z, J W)+R(X, J Z, Y, J W)+R(X, J W, Y, J Z) \text {. }
$$

From 3.3 and the usual symmetries of $R$ we obtain that $Q$ is totally symmetric. But $Q(X, X, X, X)=3 \bar{H}(X)$; whence $Q=0$. Now, since $\nabla J=0$, it is immediate to prove that

$$
R(X, Y, X, Y)=R(l X, l Y, l X, l Y)+R\left(l_{3} X, l_{3} Y, l_{3} X, l_{3} Y\right) .
$$

Since $J^{2} l=l, J^{2} l_{3}=-l_{3}$, the same technique of the Kaehler case (see [5]) leads to

$$
\begin{aligned}
& R(l X, l Y, l X, l Y)=0, \\
& R\left(l_{3} X, l_{3} Y, l_{3} X, l_{3} Y\right)=0 .
\end{aligned}
$$

Thus, $R(X, Y, X, Y)=0$, whence $R=0$.

3.5. Corollary. Let $(M, J, g)$ be an e-( $\left.J^{4}=1\right)$-Kaehler manifold. If $\tilde{R}$ is a $(0,4)$ tensor field having the usual symmetries of $R$ and also the one given in 3.3, and if

$$
\tilde{R}(X, J X, X, J X)=\bar{H}(X)
$$

for all $X \in T M$, then $\tilde{R}=R$. 
We now define the $(0,4)$ tensor field $R^{\prime}$ on $M$ by

$$
\begin{aligned}
R^{\prime}(X, Y, Z, W)=\frac{1}{4}\{ & g(X, l Z) g(Y, l W)-g(X, l W) g(Y, l Z) \\
& -g(X, J l Z) g(Y, J l W)+g(X, J l W) g(Y, J l Z) \\
& -2 g(X, J l Y) g(Z, J l W)+g\left(X, l_{3} Z\right) g\left(Y, l_{3} W\right) \\
& -g\left(X, l_{3} W\right) g\left(Y, l_{3} Z\right)+g\left(X, J l_{3} Z\right) g\left(Y, J l_{3} W\right) \\
& -g\left(X, J l_{3} W\right) g\left(Y, J l_{3} Z\right) \\
& \left.+2 g\left(X, J l_{3} Y\right) g\left(Z, J l_{3} W\right)\right\},
\end{aligned}
$$

whose properties are given in the following

3.6. Proposition. The field $R^{\prime}$ has the usual symmetries of the Riemann-Christoffel tensor and also the symmetry of Proposition 3.3. The following relations hold:

$$
\begin{aligned}
& R^{\prime}(X, Y, X, Y) \\
& =\frac{1}{4}\left\{g(X, l X) g(Y, l Y)-g(X, l Y)^{2}-3 g(X, J l Y)^{2}\right. \\
& \left.+g\left(X, l_{3} X\right) g\left(Y, l_{3} Y\right)-g\left(X, l_{3} Y\right)^{2}+3 g\left(X, J l_{3} Y\right)^{2}\right\} ; \\
& R^{\prime}(X, J X, X, J X)=g\left(X, l_{3} X\right)^{2}-g(X, l X)^{2} \text {. }
\end{aligned}
$$

Proof. Immediate.

From this, we deduce the

3.7. Proposition. Let $(M, J, g)$ be an $e-\left(J^{4}=1\right)$-Kaehler manifold such that for each $x \in M$, there exists $c_{x} \in \mathbf{R}$ satisfying $H(X)=c_{x}$ for every $X \in T_{x} M$ such that $g(X, X) g(J X, J X) \neq 0$. Then $R=c R^{\prime}$, where $c$ is the function defined by $x \rightarrow c_{x}$. And conversely.

Proof. Since $g(X, X) g(J X, J X)=g\left(X, l_{3} X\right)^{2}-g(X, l X)^{2}$, we deduce from 3.6 that

$$
\bar{H}(X)=c R^{\prime}(X, J X, X, J X) .
$$

Hence $\left(R-c R^{\prime}\right)(X, J X, X, J X)=0$ for all $X$ such that

$$
g(X, X) g(J X, J X) \neq 0 .
$$

Now, if $X$ verifies $g(X, X) g(J X, J X)=0$, then we can choose a sequence $\left\{X_{m}\right\}$ such that $X_{m} \rightarrow X$ and

$$
g\left(X_{m}, X_{m}\right) g\left(J X_{m}, J X_{m}\right) \neq 0 .
$$

In fact, $g(X, X) g(J X, J X)$ is a polynomial in the components of $X$ whose set of zeros does not contain any open subset. Since 
$\left(R-c R^{\prime}\right)\left(X_{m}, J X_{m}, X_{m}, J X_{m}\right)=0$ for each index $m$, we have by continuity that $\left(R-c R^{\prime}\right)(X, J X, X, J X)=0$. Then, by 3.5 we have $R=c R^{\prime}$. The converse is obvious.

If the $e-\left(J^{4}=1\right)$-Kaehler manifold $(M, J, g)$ satisfies the conditions of the above proposition, we say that it is of constant J-sectional curvature $c$. We have the following result of Schur type.

3.8. ThEOREM. Let $(M, J, g)$ be an $e-\left(J^{4}=1\right)$-Kaehler manifold of constant $J$-sectional curvature $c$. If $r, s>0$, or if $r=0, s>1$, or if $r>1, s=0$, then $c$ is a constant function.

Proof. We first choose an orthogonal basis of $T_{x} M,\left\{U_{i}, V_{i}, W_{j}\right.$, $\left.J W_{j}\right\}(i=1, \ldots, r ; j=1, \ldots, s)$ such that $\left\{U_{i}, V_{i}\right\}$ is a basis of $l T_{x} M,\left\{W_{j}, J W_{j}\right\}$ is a basis of $l_{3} T_{x} M, g\left(U_{i}, U_{j}\right)=-\delta_{i j}, g\left(V_{i}, V_{j}\right)=$ $g\left(W_{i}, W_{j}\right)=g\left(J W_{i}, J W_{j}\right)=\delta_{i j},(i, j=1, \ldots, r$ or $i, j=1, \ldots, s)$. If $S$ is the Ricci tensor field, we have

$$
\begin{aligned}
S(X, Y)= & -\sum_{i=1}^{r} R\left(U_{i}, X, U_{i}, Y\right)+\sum_{i=1}^{r} R\left(V_{i}, X, V_{i}, Y\right) \\
& +\sum_{i=1}^{s} R\left(W_{i}, X, W_{i}, Y\right)+\sum_{i=1}^{s} R\left(J W_{i}, X, J W_{i}, Y\right) .
\end{aligned}
$$

From this, and applying 3.7, we obtain after a calculation

$$
S(X, Y)=\frac{c}{2}\left\{g(X, Y)+r g(X, l Y)+s g\left(X, l_{3} Y\right)\right\} \text {. }
$$

Since $R=c R^{\prime}$ and $\nabla R^{\prime}=0$, we have $\nabla_{X} R=X(c) R^{\prime}$. Now, if $\left\{e_{i}\right\}$ is any orthonormal basis of $T_{x} M$ in the sense that $g\left(e_{i}, e_{j}\right)=a_{i} \delta_{i j}$ with $a_{i} \in\{-1,1\}$, we have by direct application of the second Bianchi identity

$$
\sum_{i}\left\{X(c) S\left(a_{i} e_{i}, e_{i}\right)-2 e_{i}(c) S\left(X, a_{i} e_{i}\right)\right\}=0
$$

Now,

$$
\sum_{i} S\left(X, a_{i} e_{i}\right) e_{i}=\frac{c}{2}\left(X+r l X+s l_{3} X\right)
$$

because of (1). Therefore, from (2):

$$
\left(r^{2}+s^{2}+r+s-1\right) X\left(c^{2}\right)-r l X\left(c^{2}\right)-s l_{3} X\left(c^{2}\right)=0 .
$$

If $X=l X$, then

$$
\left(r^{2}+s^{2}+s-1\right) X\left(c^{2}\right)=0
$$


If $X=l_{3} X$, then

$$
\left(r^{2}+s^{2}+r-1\right) X\left(c^{2}\right)=0 .
$$

Then, if $r, s>0$, or if $s=0, r>1$, or if $s>1, r=0$, we obtain

$$
X\left(c^{2}\right)=l X\left(c^{2}\right)+l_{3} X\left(c^{2}\right)=0 .
$$

Thus $c^{2}$, and therefore $c$, are constants.

In the conditions of the preceding Theorem, the scalar curvature is given by the function

$$
\rho=c\{r(r+1)+s(s+1)\} .
$$

Thus, if $r=s=1$, we have $\rho=4 c$.

3.9. TheOREM. Let $(M, J, g)$ be an e-( $\left.J^{4}=1\right)$-Kaehler manifold of constant $J$-sectional curvature $c$. Then:

(i) if $X, Y \in l_{3} T_{X} M$ we have

$$
\begin{aligned}
c / 4 & \leq K(X, Y) \leq c, & & \text { if } c>0 ; \\
c & \leq K(X, Y) \leq c / 4, & & \text { if } c<0 ;
\end{aligned}
$$

(ii) Let us denote by $K_{L}$ the restriction of $K$ to the planes of ITM. Then:

$$
\begin{aligned}
& K_{L}(X, Y)=c \quad \text { if } r=1 \\
& K_{L} \text { is unbounded if } r>1, c \neq 0 .
\end{aligned}
$$

Proof. (i) The restriction of $g$ to $l_{3} T M$ is Riemannian. Then if we choose $\{X, Y\}$ orthonormal, we have:

$$
K(X, Y)=\frac{c}{4}\left(1+3 g(X, J Y)^{2}\right)=\frac{c}{4}\left(1+3 \cos ^{2} \alpha\right),
$$

where $\alpha$ is the angle between the plane $\{X, Y\}$ and the plane $\{J X, J Y\}$, and the claim is obvious;

(ii) If $r=1$ we can choose a basis $\{X, J X\}$ of $l T_{x} M$; thus $K(X, J X)=$ $H(X)=c$. Now assume that $c \neq 0, r>1$. Let $\left(U_{1}, V_{1}\right) \in l_{1} T_{x} M$, $\left(U_{2}, V_{2}\right) \in l_{2} T_{x} M$ be such that $g\left(U_{1}, U_{2}\right)=g\left(V_{1}, V_{2}\right)=1, g\left(U_{1}, V_{2}\right)=$ $g\left(U_{2}, V_{1}\right)=0$. Here, $l_{1}$ and $l_{2}$ are the projectors on $l T_{x} M$ given by the eigenvalues +1 and -1 of $J \mid l T_{x} M$. We take first

$$
\begin{aligned}
& X=U_{1}+V_{1}-U_{2}+\frac{1}{2} V_{2}, \\
& Y=U_{1}+(1-\lambda) V_{1}+\frac{\lambda}{2} U_{2}+\frac{1}{2} V_{2} .
\end{aligned}
$$


Then $g(X, X)=-1, g(Y, Y)=1, g(X, J Y)=-(1+\lambda), g(X, Y)=0$. Hence $K(X, Y)=(c / 4)\left(1+3(1+\lambda)^{2}\right)$.

Now, we take

$$
\begin{aligned}
& X=U_{1}+V_{1}+U_{2}-\frac{1}{2} V_{2}, \\
& Y=\frac{\lambda^{2}}{2} U_{1}+\left(\lambda^{2}-\lambda+1\right) V_{1}-\lambda U_{2}+\frac{\lambda+1}{2} V_{2} .
\end{aligned}
$$

Then $g(X, X)=g(Y, Y)=1, g(X, Y)=0, g(X, J Y)=\lambda-1$. Hence $K(X, Y)=(c / 4)\left(1-3(\lambda-1)^{2}\right)$, and this proves our claim.

3.10. Definition. We say that two metric $\left(J^{4}=1\right)$-manifolds $(M, J, g)$ and $\left(M^{\prime}, J^{\prime}, g^{\prime}\right)$ are $J$-isometric if there exists an isometry $f: M \rightarrow M^{\prime}$ such that $f_{*} \circ J=J^{\prime} \circ f_{*}$.

It is clear that in the case of almost Hermitian manifolds this definition is the usual one for holomorphically isometric manifolds. Also we can generalize Theorem 7.9 of [5], Vol. II to obtain

3.11. Proposition. Two complete, connected and simply connected $e-\left(J^{4}=1\right)$-Kaehler manifolds of constant and equal $J$-sectional curvature $c$ are $J$-isometric (we assume that $c$ is a constant function).

Proof. It is enough to apply Proposition 2.5 which furnishes the expression of $R$ in terms of $J$ and $g$ in the case of spaces of constant $J$-sectional curvature.

4. The models of constant $J$-sectional curvature. Let $(M, J, g)$ be an $e-\left(J^{4}=1\right)$-Kaehler manifold; then it is locally the product of a paraKaehler manifold and a Kaehler manifold. Since the latter, in the case of constant holomorphic sectional curvature, is well known (see [5]), we are interested in the para-Kaehler case.

Thus, let $(M, J, g)$ be a para-Kaehler space of constant $J$-sectional curvature $c$, and assume $r>1$. Then $c$ is a constant function. We have $J^{2}=1$ and $g(X, J Y)+g(J X, Y)=0$.

Let $x_{0} \in M$, and $\left\{e_{i}, e_{i+r}\right\}$ be an orthonormal basis of $T_{x_{0}} M$, i.e.:

$$
\begin{gathered}
g\left(e_{i}, e_{j}\right)=-\delta_{i j}, \quad g\left(e_{i+r}, e_{j+r}\right)=\delta_{i j}, \quad g\left(e_{i}, e_{j+r}\right)=0, \\
J e_{i}=e_{i+r}, \quad J e_{i+r}=e_{i} .
\end{gathered}
$$

If we put $R_{A B C D}=R\left(e_{A}, e_{B}, e_{C}, e_{D}\right), A, B, C, D \in\{1, \ldots, 2 r\}$, then

$$
\begin{aligned}
R_{A B C D}=\frac{c}{4}\left(g_{A C} g_{B D}-\right. & g_{A D} g_{B C}-g_{A C \pm r} g_{B D \pm r} \\
& \left.+g_{A D \pm r} g_{B C \pm r}-2 g_{A B \pm r} g_{C D \pm r}\right)
\end{aligned}
$$


where

$$
E \pm r= \begin{cases}E+r & \text { if } 1 \leq E \leq r \\ E-r & \text { if } r+1 \leq E \leq 2 r\end{cases}
$$

Prvanovic [9] obtains this expression in a different way.

Now, we apply the structural equations in polar coordinates in order to obtain $g$ and $J$ in these coordinates (see [1], [12]).

For doing that, let $I$ be an interval of $\mathbf{R}$ containing 0 and $1, U$ a neighbourhood of 0 in $T_{x_{0}} M$ and $V$ a neighbourhood of $x_{0}$ in $M$ such that exp: $U \rightarrow V$ is a diffeomorphism and such that the map $\Phi: I \times U \rightarrow M$ given by $\Phi(t, X)=\exp (t X)$ is well defined. If $\left\{\gamma^{A}\right\}$ is the dual of $\left\{e_{A}\right\}$, we have coordinates $\left(t, t^{A}\right)$ on $I \times U$ given by $t\left(t_{0}, X\right)=t_{0}, t^{A}\left(t_{0}, X\right)=\gamma^{A}(X)$.

By parallel transport of $\left\{e_{A}\right\}$ along the geodesics starting at $x_{0}$ we obtain a frame $\left\{e_{A}\right\}$ on $V$ with dual $\left\{\gamma^{A}\right\}$. If we define the 1 -forms $\vartheta^{A}$ on $I \times U$ by

$$
\vartheta^{A}=\phi^{*} \gamma^{A}-t^{A} d t
$$

then $i(\partial / \partial t) \vartheta^{A}=0$, and we have the conditions

$$
\begin{gathered}
\vartheta_{(0, X)}^{A}=0,\left.\quad \frac{\partial \vartheta^{A}}{\partial t}\right|_{(0, X)}=\left.d t^{A}\right|_{(0, X)}, \\
\frac{\partial^{2} \vartheta^{A}}{\partial t^{2}}=\left(R_{B C D}^{A} \circ \phi\right) t^{B} t^{C} \vartheta^{D} .
\end{gathered}
$$

Thus

$$
\begin{aligned}
& \frac{\partial^{2} \vartheta^{i}}{\partial t^{2}}=-\left(R_{i B C D} \circ \phi\right) t^{B} t^{C} \vartheta^{D} \\
& =-\frac{c}{4}\left\{t^{j}\left(t^{i} \vartheta^{j}-t^{j} \vartheta^{i}-t^{i+r} \vartheta^{j+r}+t^{j+r} \vartheta^{i+r}\right)\right. \\
& +t^{j+r}\left(t^{j+r} \vartheta^{i}-t^{i} \vartheta^{j+r}+t^{i+r} \vartheta^{j}-t^{j} \vartheta^{i+r}\right) \\
& \left.+2 t^{i+r}\left(t^{j+r} \vartheta^{j}-t^{j} \vartheta^{j+r}\right)\right\} \\
& \frac{\partial^{2} \vartheta^{i+r}}{\partial t^{2}}=\left(R_{i+r B C D} \circ \phi\right) t^{B} t^{C} \vartheta^{D} \\
& =-\frac{c}{4}\left\{t^{j+r}\left(t^{i} \vartheta^{j}-t^{j} \vartheta^{i}-t^{i+r} \vartheta^{j+r}+t^{j+r} \vartheta^{i+r}\right)\right. \\
& +t^{j}\left(t^{j+r} \vartheta^{i}-t^{i} \vartheta^{j+r}+t^{i+r} \vartheta^{j}-t^{j} \vartheta^{i+r}\right) \\
& \left.+2 t^{i}\left(t^{j+r} \vartheta^{j}-t^{j} \vartheta^{j+r}\right)\right\} .
\end{aligned}
$$

To simplify this, we introduce on $I \times U$ new coordinates $\left\{a^{i}, b^{i}\right\}$ and new 1-forms $\mu^{i}, \nu^{i}$ by:

$$
a^{i}=\frac{t^{i}+t^{i+r}}{\sqrt{2}}, \quad b^{i}=\frac{t^{i}-t^{i+r}}{\sqrt{2}}, \quad \mu^{i}=\frac{\vartheta^{i}+\vartheta^{i+r}}{\sqrt{2}}, \quad \nu^{i}=\frac{\vartheta^{i}-\vartheta^{i+r}}{\sqrt{2}} .
$$


Then

$$
\begin{aligned}
\frac{\partial^{2} \mu^{i}}{\partial t^{2}} & =\frac{c}{4}\left(a^{j} b^{j} \mu^{i}+a^{i} b^{j} \mu^{j}-2 a^{i} a^{j} \nu^{j}\right), \\
\frac{\partial^{2} \nu^{i}}{\partial t^{2}} & =\frac{c}{4}\left(a^{j} b^{j} \nu^{i}+b^{i} a^{j} \nu^{j}-2 b^{i} b^{j} \mu^{j}\right) .
\end{aligned}
$$

By putting $\langle a, b\rangle=a^{j} b^{j}$, etc., this can be written

$$
\begin{aligned}
& \frac{\partial^{2} \mu}{\partial t^{2}}=\frac{c}{4}(\langle a, b\rangle \mu+\langle b, \mu\rangle a-2\langle a, \nu\rangle a), \\
& \frac{\partial^{2} \nu}{\partial t^{2}}=\frac{c}{4}(\langle a, b\rangle \nu+\langle a, \nu\rangle b-2\langle b, \mu\rangle a) .
\end{aligned}
$$

If we put $\rho^{2}=-\frac{1}{2} c\langle a, b\rangle$, these equations read

$$
\begin{aligned}
& \frac{\partial^{2} \mu}{\partial t^{2}}+\rho^{2} \mu=-\frac{\rho^{2}}{\langle a, b\rangle}\langle b, \mu\rangle a+\frac{2 \rho^{2}}{\langle a, b\rangle}\langle a, \nu\rangle a, \\
& \frac{\partial^{2} \nu}{\partial t^{2}}+\rho^{2} \nu=-\frac{\rho^{2}}{\langle a, b\rangle}\langle a, \nu\rangle b+\frac{2 \rho^{2}}{\langle a, b\rangle}\langle b, \mu\rangle b .
\end{aligned}
$$

If we multiply (3) by $b$ and (4) by $a$, we obtain

$$
\begin{aligned}
& \left\langle b, \frac{\partial^{2} \mu}{\partial t^{2}}\right\rangle+\rho^{2}\langle b, \mu\rangle=-\rho^{2}\langle b, \mu\rangle+2 \rho^{2}\langle a, \nu\rangle, \\
& \left\langle a, \frac{\partial^{2} \nu}{\partial t^{2}}\right\rangle+\rho^{2}\langle a, \nu\rangle=-\rho^{2}\langle a, \nu\rangle+2 \rho^{2}\langle b, \mu\rangle .
\end{aligned}
$$

By adding and subtracting (5) and (6), we get

$$
\begin{aligned}
& \frac{\partial^{2}}{\partial t^{2}}(\langle b, \mu\rangle+\langle a, \nu\rangle)=0, \\
& \frac{\partial^{2}}{\partial t^{2}}(\langle b, \mu\rangle-\langle a, \nu\rangle)+4 \rho^{2}(\langle b, \mu\rangle-\langle a, \nu\rangle)=0,
\end{aligned}
$$

with the initial conditions

$$
\mu_{(0)}=\nu_{(0)}=0,\left.\quad \frac{\partial \mu}{\partial t}\right|_{0}=d a,\left.\quad \frac{\partial \nu}{\partial t}\right|_{0}=d b .
$$

The solution of the system (7), (8), (9) is obviously

$$
\begin{aligned}
& \langle b, \mu\rangle=\frac{\langle b, d a\rangle-\langle a, d b\rangle}{4 \rho} \sin 2 \rho t+\frac{1}{2}(\langle b, d a\rangle+\langle a, d b\rangle) t, \\
& \langle a, \nu\rangle=\frac{\langle a, d b\rangle-\langle b, d a\rangle}{4 \rho} \sin 2 \rho t+\frac{1}{2}(\langle b, d a\rangle+\langle a, d b\rangle) t .
\end{aligned}
$$


By substitution in (3), we get

$$
\begin{aligned}
\frac{\partial^{2} \mu}{\partial t^{2}}+\rho^{2} \mu= & -\frac{3 \rho}{4\langle a, b\rangle}(\langle b, d a\rangle-\langle a, d b\rangle)(\sin 2 \rho t) a \\
& +\frac{\rho^{2}}{2\langle a, b\rangle}(\langle b, d a\rangle+\langle a, d b\rangle) t a .
\end{aligned}
$$

It we call

$$
\eta=\mu-\frac{1}{2\langle a, b\rangle}(\langle b, d a\rangle+\langle a, d b\rangle) t a,
$$

then this equation reads

$$
\frac{\partial^{2} \eta}{\partial t^{2}}+\rho^{2} \eta=-\frac{3 \rho}{4\langle a, b\rangle}(\langle b, d a\rangle-\langle a, d b\rangle)(\sin 2 \rho t) a .
$$

We seek a particular solution of the type $\eta=(D / \rho\langle a, b\rangle)(\sin 2 \rho t) a$. Then we get the condition

$$
D=\frac{1}{4}(\langle b, d a\rangle-\langle a, d b\rangle) .
$$

Thus the solution is

$$
\begin{aligned}
\mu= & \frac{1}{2\langle a, b\rangle}(\langle b, d a\rangle+\langle a, d b\rangle) t a+\frac{A}{\langle a, b\rangle} \sin (\rho t) \\
& +\frac{\langle b, d a\rangle-\langle a, d b\rangle}{4 \rho\langle a, b\rangle} \sin (2 \rho t) a .
\end{aligned}
$$

And the initial conditions imply

$$
\begin{aligned}
\mu= & \frac{\langle a, b\rangle d a-\langle b, d a\rangle a}{\langle a, b\rangle \rho} \sin \rho t+\frac{(\langle b, d a\rangle-\langle a, d b\rangle) a}{4\langle a, b\rangle \rho} \sin 2 \rho t \\
& +\frac{\langle b, d a\rangle+\langle a, d b\rangle}{2\langle a, b\rangle} d t, \\
\nu= & \frac{\langle a, b\rangle d b-\langle a, d b\rangle b}{\langle a, b\rangle \rho} \sin \rho t+\frac{(\langle a, d b\rangle-\langle b, d a\rangle) b}{4\langle a, b\rangle \rho} \sin 2 \rho t \\
& +\frac{\langle b, d a\rangle+\langle a, d b\rangle}{2\langle a, b\rangle} b t .
\end{aligned}
$$

Now, we define 1 -forms $\alpha^{i}, \beta^{i}(i=1, \ldots, r)$ on $U$ by

$$
\alpha^{i}=\mu^{i}(1), \quad \beta^{i}=\nu^{i}(1),
$$

and also define a metric on $U, \tilde{g}$, by

$$
\tilde{g}=-\alpha^{i} \otimes \beta^{i}-\beta^{i} \otimes \alpha^{i},
$$

and a tensor field $\tilde{J}$ on $U$ by

$$
\tilde{J}=u_{i} \otimes \alpha^{i}-v_{i} \otimes \beta^{i},
$$


where $\left\{u_{i}, v_{i}\right\}$ is the dual of $\left\{\alpha^{i}, \beta^{i}\right\}$. Then, the map exp: $U \rightarrow V$ is a $J$-isometry as it is easily checked. Thus, we compute $\tilde{g}$ and $\tilde{J}$. First we have

$$
\begin{aligned}
& \alpha^{i}=\frac{\sin \rho}{\rho} d a^{i}+\frac{\sin 2 \rho-4 \sin \rho+2 \rho}{4\langle a, b\rangle \rho} b^{k} a^{i} d a^{k}+\frac{2 \rho-\sin 2 \rho}{4\langle a, b\rangle \rho} a^{k} a^{i} d b^{k} \\
& \beta^{i}=\frac{\sin \rho}{\rho} d b^{i}+\frac{\sin 2 \rho-4 \sin \rho+2 \rho}{4\langle a, b\rangle \rho} a^{k} b^{i} d b^{k}+\frac{2 \rho-\sin 2 \rho}{4\langle a, b\rangle \rho} b^{k} b^{i} d a^{k} .
\end{aligned}
$$

Therefore, by substitution

$$
\begin{aligned}
\tilde{g}=-\{ & \frac{\sin ^{2} \rho}{\rho^{2}}\left(d a^{i} \otimes d b^{i}+d b^{i} \otimes d a^{i}\right) \\
& +\frac{4 \rho^{2}-\sin ^{2} 2 \rho}{8\langle a, b\rangle \rho^{2}}\left(a^{i} a^{k} d b^{i} \otimes d b^{k}+b^{i} b^{k} d a^{i} \otimes d a^{k}\right) \\
& \left.+\frac{4 \rho^{2}+\sin ^{2} 2 \rho-8 \sin ^{2} \rho}{8\langle a, b\rangle \rho^{2}} a^{i} b^{k}\left(d b^{i} \otimes d a^{k}+d a^{k} \otimes d b^{i}\right)\right\} .
\end{aligned}
$$

Note that even in the case of $\rho^{2}<0$, the above result is a real tensor field, and it is $C^{\infty}$ also in the points where $\rho=0$.

As for the dual base, we have

$$
\begin{aligned}
u_{j}= & \frac{\rho}{\sin \rho} \frac{\partial}{\partial a^{j}}+\frac{\sin 2 \rho-2 \rho}{2\langle a, b\rangle \sin 2 \rho} b^{j} b^{l} \frac{\partial}{\partial b^{l}} \\
& +\frac{\sin \rho \sin 2 \rho+2 \rho \sin \rho-2 \rho \sin 2 \rho}{2\langle a, b\rangle \sin \rho \sin 2 \rho} b^{j} a^{l} \frac{\partial}{\partial a^{l}}, \\
v_{j}= & \frac{\rho}{\sin \rho} \frac{\partial}{\partial b^{j}}+\frac{\sin 2 \rho-2 \rho}{2\langle a, b\rangle \sin 2 \rho} a^{j} a^{l} \frac{\partial}{\partial a^{l}} \\
& +\frac{\sin \rho \sin 2 \rho+2 \rho \sin \rho-2 \rho \sin 2 \rho}{2\langle a, b\rangle \sin \rho \sin 2 \rho} a^{j} b^{l} \frac{\partial}{\partial b^{l}} .
\end{aligned}
$$

Therefore, we have by substitution

$$
\begin{aligned}
\tilde{J}= & \frac{\partial}{\partial a^{i}} \otimes d a^{i}-\frac{\partial}{\partial b^{i}} \otimes d b^{i} \\
& +\frac{(2 \rho-\sin 2 \rho)^{2}}{4\langle a, b\rangle \rho \sin 2 \rho} a^{i} b^{k}\left(\frac{\partial}{\partial a^{i}} \otimes d a^{k}-\frac{\partial}{\partial b^{k}} \otimes d b^{i}\right) \\
& +\frac{4 \rho^{2}-\sin ^{2} 2 \rho}{4\langle a, b\rangle \rho \sin 2 \rho}\left(a^{i} a^{k} \frac{\partial}{\partial a^{i}} \otimes d b^{k}-b^{i} b^{k} \frac{\partial}{\partial b^{i}} \otimes d a^{k}\right) .
\end{aligned}
$$

The expression of $\tilde{g}$ and $\tilde{j}$ give the space form in normal coordinates for the para-Kaehler manifolds of constant $J$-sectional curvature and $r>1$. If $r=1$, we have automatically $N=0, d F=0, \nabla J=0$, (cfr. 
3.1) and the space is of constant $J$-sectional curvature $c$, but $c$ may not be a constant. However if $c$ were a constant, the above formulae for normal coordinates are also valid. Thus, we will say in the following that an almost para-Hermitian manifold with $r=1$ is a para-Kaehler manifold of constant $J$-sectional curvature if the above function $c$ is constant.

Now, let $B$ be the vector space $\mathbf{R}^{2}$ with the product $(a, b)\left(a^{\prime}, b^{\prime}\right)=$ $\left(a a^{\prime}, b b^{\prime}\right)$; then $B$ is a commutative algebra. If we define the conjugate $\bar{w}$ of an element $w=(a, b) \in B$ by $\bar{w}=(b, a)$, then an element $w \in B$ is real if $w=\bar{w}$, and is invertible if $w \bar{w} \neq 0$. We put $B_{+}=\{(a, b) \in$ $B \mid a>0, b>0\}$; then $B_{+}$is a Lie group. Let

$$
B_{0}^{r+1}=\left\{z=\left(z^{\alpha}\right) \in B^{r+1} \mid\langle z, \bar{z}\rangle>0\right\},
$$

where

$$
\langle z, \bar{z}\rangle=\sum_{\alpha=0}^{r} z^{\alpha} \bar{z}^{\alpha} .
$$

We denote by $\mathfrak{g l}(B ; r+1)$ the algebra of $(r+1) \times(r+1)$-matrices with elements in $B$. Then $\mathfrak{g l}(B ; r+1)=\mathfrak{g l}(\mathbf{R} ; r+1) \times \mathfrak{g l}(\mathbf{R} ; r+1)$. We have the Lie group

$$
U(B ; r+1)=\left\{Z \in \mathfrak{g l}(B ; r+1) \mid\langle Z z, \bar{Z} \bar{z}\rangle=\langle z, \bar{z}\rangle \text { for all } z \in B^{r+1}\right\} .
$$

Let $P_{r}(B)$ be the quotient of $B_{0}^{r+1}$ under the equivalence given by $\left(z^{\alpha}\right)=\left(q z^{\alpha}\right), q \in B_{+}$. Then, if $\pi: B_{0}^{r+1} \rightarrow P_{r}(B)$ is the natural projection, we can identify $\pi(z)$ with the unique element $w=q z$ such that $\langle w, \bar{w}\rangle=1,\langle w, w\rangle=\langle\bar{w}, \bar{w}\rangle$, where $q=(a, b) \in B_{+}$. Indeed, if $z=\left(z^{\alpha}\right)=\left(\left(u^{\alpha}, v^{\alpha}\right)\right)$, we have

$$
\begin{gathered}
\langle w, \bar{w}\rangle=(a b\langle u, v\rangle, a b\langle u, v\rangle), \quad\langle w, w\rangle=\left(a^{2}\langle u, u\rangle, b^{2}\langle v, v\rangle\right), \\
\langle\bar{w}, \bar{w}\rangle=\left(b^{2}\langle v, v\rangle, a^{2}\langle u, u\rangle\right) .
\end{gathered}
$$

Then

$$
a=\frac{\langle v, v\rangle^{1 / 4}}{\langle u, u\rangle^{1 / 4}\langle u, v\rangle^{1 / 2}}, \quad b=\frac{\langle u, u\rangle^{1 / 4}}{\langle v, v\rangle^{1 / 4}\langle u, v\rangle^{1 / 2}}
$$

Thus

$$
P_{r}(B) \simeq\left\{(u, v) \in \mathbf{R}^{r+1} \times \mathbf{R}^{r+1} \mid\langle u, u\rangle=\langle v, v\rangle,\langle u, v\rangle=1\right\} .
$$

Since $Z(q z)=q Z(z)$ for all $Z \in U(B ; r+1), z \in B_{0}^{r+1}, q \in B_{+}$, it is clear that the action of $U(B ; r+1)$ pass to the quotient $P_{r}(B)$. 
4.1. Proposition. $P_{r}(B)$ is diffeomorphic to $T S^{r}$; therefore it is connected and if $r>1$ it is simply connected. The group $U(B ; r+1)$ acts transitively on $P_{r}(B)$.

Proof. We consider the map $\varphi: P_{r}(B) \rightarrow T S^{r}$ given by $\varphi(u, v)=$ $\left(\|u+v\|^{-1}(u+v), u-v\right)$. Since $\langle u, u\rangle=\langle v, v\rangle$, we have that $\left\langle\|u+v\|^{-1}(u+v), u-v\right\rangle=0$, then $u-v$ can be considered as a vector tangent to $S^{r}$ at the point $\|u+v\|^{-1}(u+v)$. It is immediate to prove that $\varphi$ is a diffeomorphism. Now, let $(u, v) \in P_{r}(B)$; if $\left\{e_{\alpha}\right\}$ is the canonical basis of $\mathbf{R}^{r+1}$ and $\left\{\vartheta^{\alpha}\right\}$ its dual, let $\gamma^{i}(i=1, \ldots, r)$ be a linearly independent set of 1 -forms such that $\gamma^{i}(u)=0$. If $\gamma^{i}=\gamma_{\alpha}^{i} \vartheta^{\alpha}$, and $v=v^{\alpha} e_{\alpha}$, we define $P \in \mathrm{Gl}(r+1 ; \mathbf{R})$ by putting $\vartheta^{0}\left(P e_{\alpha}\right)=v^{\alpha}$, $\vartheta^{i}\left(P e_{\alpha}\right)=\gamma_{\alpha}^{i}$. Then

$$
\begin{gathered}
P u=u^{\alpha} P e_{\alpha}=u^{\alpha} \vartheta^{0}\left(P e_{\alpha}\right) e_{0}+u^{\alpha} \vartheta^{i}\left(P e_{\alpha}\right) e_{i}=u^{\alpha} v^{\alpha} e_{0}+u^{\alpha} \gamma_{\alpha}^{i} e_{i}=e_{0} \\
{ }^{t} P e_{0}=\vartheta^{\alpha}\left({ }^{t} P e_{0}\right) e_{\alpha}=\vartheta^{0}\left(P e_{\alpha}\right) e_{\alpha}=v^{\alpha} e_{\alpha}=v .
\end{gathered}
$$

Therefore $\left(P,{ }^{t} P^{-1}\right)(u, v)=\left(e_{0}, e_{0}\right)$ and since $\left(P,{ }^{t} P^{-1}\right) \in U(B ; r+1)$, it is clear that $U(B ; r+1)$ acts transitively on $P_{r}(B)$.

We consider on $B_{0}^{r+1}$ the covariant tensor field $(0 \neq c \in \mathbf{R})$ :

$$
\begin{aligned}
\tilde{g}=\frac{2}{c\langle u, v\rangle} & \left\{d u^{\alpha} \otimes d v^{\alpha}+d v^{\alpha} \otimes d u^{\alpha}\right. \\
& \left.-\frac{1}{\langle u, v\rangle} u^{\alpha} v^{\beta}\left(d v^{\alpha} \otimes d u^{\beta}+d u^{\beta} \otimes d v^{\alpha}\right)\right\} .
\end{aligned}
$$

Then $\tilde{g}$ is invariant by $U(B ; r+1)$ as it is easily proved. If $i: P_{r}(B) \rightarrow$ $B_{0}^{r+1}$ is the inclusion, we have by direct computation that $(i \cdot \pi)^{*} \tilde{g}=\tilde{g}$. Hence, the tensor field $g=i^{*} \tilde{g}$, which is a pseudo-Riemannian metric on $P_{r}(B)$, is also invariant by $U(B ; r+1)$. We have for $P_{r}(B)$ the charts $\left(\varphi^{\alpha}, U_{\alpha}^{ \pm}\right)$, where

$$
\begin{aligned}
& \left.U_{\alpha}^{+}=\{(u, v)\} \in P_{r}(B) \mid u^{\alpha}>0, v^{\alpha}>0\right\}, \\
& \left.U_{\alpha}^{-}=\{(u, v)\} \in P_{r}(B) \mid u^{\alpha}<0, v^{\alpha}<0\right\}
\end{aligned}
$$

and

$$
\varphi^{\alpha}(u, v)=\left(\frac{u^{0}}{u^{\alpha}}, \ldots, \frac{\hat{u}^{\alpha}}{u^{\alpha}}, \ldots, \frac{u^{r}}{u^{\alpha}} ; \frac{v^{0}}{v^{\alpha}}, \ldots, \frac{\hat{v}^{\alpha}}{v^{\alpha}}, \ldots, \frac{v^{r}}{v^{\alpha}}\right)
$$

If we call $\left(x^{i}, y^{i}\right)$ to the coordinates of any one of these charts, say $x^{i}=u^{i} / u^{0}, y^{i}=v^{i} / v^{0}$, then by direct computation or well by an 
argument similar to the one used in [5, vol. II, p. 160], we have that (10) $g=\frac{2}{c(1+\langle x, y\rangle)}\left(d x^{i} \otimes d y^{i}+d y^{i} \otimes d x^{i}\right.$

$$
\left.-\frac{1}{1+\langle x, y\rangle} x^{i} y^{j}\left(d y^{i} \otimes d x^{j}+d x^{j} \otimes d y^{i}\right)\right) .
$$

Also, we have on $B_{0}^{r+1}$ the almost-product structure given by

$$
\tilde{J}=\frac{\partial}{\partial u^{\alpha}} \otimes d u^{\alpha}-\frac{\partial}{\partial v^{\alpha}} \otimes d v^{\alpha},
$$

and it defines an almost-product structure on $P_{r}(B), J$, by the relation $\pi_{*} \circ \tilde{J}=J \circ \pi_{*}$, which in the same chart is given by

$$
J=\frac{\partial}{\partial x^{i}} \otimes d x^{i}-\frac{\partial}{\partial y^{i}} \otimes d y^{i}
$$

Then

4.2. Theorem. $P_{r}(B)$ admits a para-Kaehler structure of constant $J$-sectional curvature $c \neq 0$ given by (10) and (11). Then $P_{r}(B)$ is connected and complete, and if $r>1$, it is also simply connected.

Proof. The 2-form $F(X, Y)=g(X, J Y)$ is given by

$$
F=\frac{2}{c(1+\langle x, y\rangle)}\left(d y^{i} \wedge d x^{i}-\frac{1}{1+\langle x, y\rangle} x^{j} d y^{j} \wedge y^{i} d x^{i}\right) .
$$

Then $d F=0$. Since evidently $N=0$, we have that $P_{r}(B)$ is a para-Kaehler manifold. Since $\nabla J=0$, we have $\nabla_{\partial / \partial x^{i}}\left(\partial / \partial y^{j}\right)=0$.

Also

$$
g\left(\frac{\partial}{\partial x^{i}}, \frac{\partial}{\partial y^{j}}\right)=\frac{2}{c} \frac{\partial}{\partial x^{i}} \frac{x^{j}}{1+\langle x, y\rangle} .
$$

Hence

$$
\begin{aligned}
g\left(\nabla_{\partial / \partial x^{i}} \frac{\partial}{\partial x^{j}}, \frac{\partial}{\partial y^{k}}\right) & =\frac{\partial}{\partial x^{i}} g\left(\frac{\partial}{\partial x^{i}}, \frac{\partial}{\partial y^{k}}\right)=\frac{2}{c} \frac{\partial^{2}}{\partial x^{i} \partial x^{j}} \frac{x^{k}}{1+\langle x, y\rangle} \\
& =-\frac{2}{c}\left\{\frac{\delta_{i k} y^{j}+\delta_{j k} y^{i}}{(1+\langle x, y\rangle)^{2}}-\frac{2 x^{k} y^{i} y^{j}}{(1+\langle x, y\rangle)^{3}}\right\}
\end{aligned}
$$

Therefore

$$
\nabla_{\partial / \partial x^{i}} \frac{\partial}{\partial x^{k}}=-\frac{1}{1+\langle x, y\rangle}\left(y^{k} \frac{\partial}{\partial x^{i}}+y^{i} \frac{\partial}{\partial x^{k}}\right) .
$$



have

And if 0 is the point of $P_{r}(B)$ with coordinates $x^{i}=y^{i}=0$, we

$$
\left(\nabla_{\partial / \partial y^{\prime}} \nabla_{\partial / \partial x^{i}} \frac{\partial}{\partial x^{k}}\right)_{0}=-\left(\delta_{k j} \frac{\partial}{\partial x^{i}}+\delta_{i j} \frac{\partial}{\partial x^{k}}\right)_{0} .
$$

Therefore

$$
\begin{aligned}
R\left(\frac{\partial}{\partial x^{i}}, \frac{\partial}{\partial y^{j}},\right. & \left.\frac{\partial}{\partial x^{k}}, \frac{\partial}{\partial y^{l}}\right)_{0}=g\left(\nabla_{\partial / \partial y^{\prime}} \nabla_{\partial / \partial x^{\prime}} \frac{\partial}{\partial x^{k}}, \frac{\partial}{\partial y^{l}}\right)_{0} \\
= & -\delta_{k j} g\left(\frac{\partial}{\partial x^{i}}, \frac{\partial}{\partial y^{l}}\right)_{0}-\delta_{i j} g\left(\frac{\partial}{\partial x^{k}}, \frac{\partial}{\partial y^{l}}\right)_{0} \\
& =-\frac{2}{c}\left(\delta_{k j} \delta_{i l}+\delta_{k l} \delta_{i j}\right), \\
R^{\prime}\left(\frac{\partial}{\partial x^{i}}, \frac{\partial}{\partial y^{j}}, \frac{\partial}{\partial x^{k}}, \frac{\partial}{\partial y^{l}}\right)_{0} & =\frac{1}{c^{2}}\left(-\delta_{i l} \delta_{j k}-\delta_{i l} \delta_{j k}-2 \delta_{i j} \delta_{k l}\right) \\
& =-\frac{2}{c^{2}}\left(\delta_{k j} \delta_{i l}+\delta_{k l} \delta_{i j}\right) .
\end{aligned}
$$

Hence $R=c R^{\prime}$ at 0 . Since $R$ and $R^{\prime}$ are invariant by $U(B ; r+1)$ we conclude that the $J$-sectional curvature is $c$, and that $\left(P_{r}(B), g\right)$ is complete.

As for the problem of finding a complete, connected and simply connected para-Kaehler manifold of constant $J$-sectional curvature in the case $r=1$, it is enough to extend the above structure on $P_{1}(B)$ up to the universal covering of $P_{1}(B)=S^{1} \times \mathbf{R}$, which is $\mathbf{R}^{2}$.

We shall study the spaces $P_{r}(B)$ as symmetric spaces in a forthcoming paper.

\section{REFERENCES}

[1] E. Cartan, Leçons sur la Géométrie des Espaces de Riemann, Gauthier-Villars, 1963.

[2] L. Graves and K. Nomizu, On sectional curvatures of indefinite metrics, Math. Ann., 232 (1978), 267-272.

[3] J. M. Hernando, P. M. Gadea and A. Montesinos, G-structures defined by tensor fields of electromagnetic type, Rend. Circ. Mat. Palermo, 34 (1985), 202-218.

[4] V. Hlavatý, Geometry of Einstein's Unified Field Theory, P. Noordhoff, 1958.

[5] S. Kobayashi and K. Nomizu, Foundations of Differential Geometry, Inters. Publ. 1963 and 1969.

[6] G. Legrand, Étude d'une généralisation des structures presque complexes sur les variétés différentiables, Rend. Circ. Mat. Palermo, Ser. 2,7 (1958), 323-354; 8 (1959), 5-48.

[7] P. Libermann, Sur le problème d'équivalence de certaines structures infinitésimales, Ann. Mat. Pura Appl., 36 (1954), 27-120. 
[8] R. S. Mishra, Structures in electromagnetic field, Tensor N.S., 30 (1976), 145156.

[9] M. Prvanović, Holomorphically projective transformations in a locally product space, Math. Balkanica, 1 (1971), 195-213.

[10] P. K. Raševski, Trudy Sem. Vektor. Tenzor. Analiz., 6 (1948), 225-248.

[11] E. Reyes, A. Montesinos and P. M. Gadea, Connections making parallel a metric $\left(J^{4}=1\right)$-structure, Anal. Sti. Univ. "Al. I. Cuza" Iasi, 28 (1982), 49-54.

[12] M. Spivak, Differential Geometry, Vol. II, Publish or Perish, 1975.

Received June 7, 1987. The second author was partially supported by project n. 120 of the CAICYT.

C.E.C.I.M.E., C.S.I.C.

28006 MADRID

SPAIN

AND

Facultad de Matematicas

46100 BURJASOT

SPAIN 



\section{PACIFIC JOURNAL OF MATHEMATICS EDITORS}

\author{
V. S. VARADARAJAN \\ (Managing Editor) \\ University of California \\ Los Angeles, CA 90024 \\ HeRbert Clemens \\ University of Utah \\ Salt Lake City, UT 84112 \\ THOMAS ENRIGHT \\ University of California, San Diego \\ La Jolla, CA 92093
}

R. FINN

Stanford University

Stanford, CA 94305

HERMANN FLASCHKA

University of Arizona

Tucson, AZ 85721

VAUGHAN F. R. JONES

University of California

Berkeley, CA 94720

STEVEN KERCKHOFF

Stanford University

Stanford, CA 94305

\section{ROBION KIRBY}

University of California

Berkeley, CA 94720

C. C. MOORE

University of California

Berkeley, CA 94720

HAROLD STARK

University of California, San Diego

La Jolla, CA 92093

\section{ASSOCIATE EDITORS}
R. ARenS
E. F. BECKENBACH
B. H. NeumanN
F. WOLF
K. YOSHIDA (1906-1982)

\section{SUPPORTING INSTITUTIONS}
UNIVERSITY OF ARIZONA
UNIVERSITY OF OREGON
UNIVERSITY OF BRITISH COLUMBIA
UNIVERSITY OF SOUTHERN CALIFORNIA
CALIFORNIA INSTITUTE OF TECHNOLOGY
STANFORD UNIVERSITY
UNIVERSITY OF CALIFORNIA
MONTANA STATE UNIVERSITY
UNIVERSITY OF HAWAII
UNIVERSITY OF NEVADA, RENO
UNIVERSITY OF TOKYO
NEW MEXICO STATE UNIVERSITY
UNIVERSITY OF UTAH
OREGON STATE UNIVERSITY
WASHINGTON STATE UNIVERSITY
UNIVERSITY OF WASHINGTON 


\section{Pacific Journal of Mathematics}

\section{Vol. 136, No. $1 \quad$ November, 1989}

Robert Archbold and Frederic W. Shultz, Characterization of $C^{*}$-algebras with continuous trace by properties of their pure states $\ldots \ldots \ldots \ldots \ldots \ldots 1$

Shu Ping Chen and Roberto Triggiani, Proof of extensions of two conjectures on structural damping for elastic systems $\ldots \ldots \ldots \ldots \ldots \ldots$

Philip Throop Church and James Timourian, A nonlinear elliptic

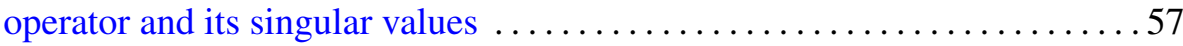

A. Gervasio Colares and Katsuei Kenmotsu, Isometric deformation of surfaces in $R^{3}$ preserving the mean curvature function $\ldots \ldots \ldots \ldots \ldots 71$

Fei Xu, A remark on spinor norms of local integral rotations. I . . . . . . . 81

Pedro Martinez Gadea and Ángel María Montesinos-Amilibia, Spaces of constant para-holomorphic sectional curvature $\ldots \ldots \ldots \ldots \ldots \ldots \ldots 5$

Guangxin Zeng, Homogeneous Stellensätze in semialgebraic geometry . . . . 103

Thomas Eric Hall, The isomorphism problem for orthodox semigroups . . . . 123

Mike Hoffman, Noncoincidence index, free group actions, and the fixed

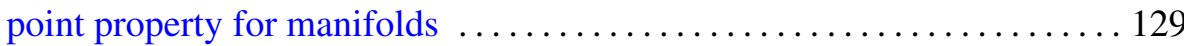

Terry Atherton Loring, The noncommutative topology of one-dimensional

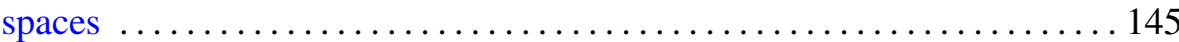

Haskell Paul Rosenthal and Alan Evan Wessel, The Krě̆ n-Mil'man property and a martingale coordinatization of certain nondentable convex sets

Yoshimi Saito, A remark on the limiting absorption principle for the reduced wave equation with two unbounded media 